\title{
MATUSEVICH, Maxim (ed.). - Africa in Russia, Russia in Africa. Three Centuries of Encounters
}

Tatiana Smirnova

\section{OpenEdition}

\section{Journals}

Édition électronique

URL : http://journals.openedition.org/etudesafricaines/20776

DOI : 10.4000/etudesafricaines.20776

ISSN : 1777-5353

\section{Éditeur}

Éditions de l'EHESS

\section{Édition imprimée}

Date de publication : 1 juillet 2017

Pagination : 465-469

ISBN : 978-2-7132-2688-5

ISSN : 0008-0055

\section{Référence électronique}

Tatiana Smirnova, « Matusevich, Maxim (ed.). - Africa in Russia, Russia in Africa. Three Centuries of Encounters », Cahiers d'études africaines [En ligne], 226 | 2017, mis en ligne le 01 avril 2017, consulté le 23 septembre 2020. URL : http://journals.openedition.org/etudesafricaines/20776 ; DOI : https:// doi.org/10.4000/etudesafricaines.20776

Ce document a été généré automatiquement le 23 septembre 2020.

(c) Cahiers d'Études africaines 


\title{
MATUSEVICH, Maxim (ed.). - Africa in Russia, Russia in Africa. Three Centuries of Encounters
}

\author{
Tatiana Smirnova
}

\section{RÉFÉRENCE}

Matusevich, Maxim (ed.). - Africa in Russia, Russia in Africa. Three Centuries of Encounters. Trenton, N.J.-Amsara, Africa World Press, 2007, 411 p., bibl., ill.

1 Africa in Russia, Russia in Africa. Three Centuries of Encounters compte peut-être parmi les travaux dont la valeur pionnière tient avant tout à l'immense tâche que s'assignent les auteurs: extraire de leur gangue exotique 300 ans de relations historiques entre la Russie et l'Afrique. Le livre montre comment ces liens demeurent d'une certaine manière prégnants et «invisibles » (p.1). L'Afrique, constituant un continent éloigné, étrange et combien exotique pour beaucoup de Russes, tout comme peut apparaitre la Russie pour nombre d'Africains, il est à craindre que l'ignorance et le manque de connaissances sur ces connexions ne donnent lieu à des mythes et des représentations plus ou moins faussés que cet ouvrage prend pour cible en cherchant à les déconstruire. Parmi ces représentations, on peut signaler, à titre d'exemple, le rôle prépondérant accordé à l'idéologie ou bien l'existence d'un plan cohérent de la politique de l'Union soviétique en Afrique. Dans cette démarche, les auteurs de ce livre collectif dirigé par Maxim Matusevich, professeur au département d'Histoire de l'Université Seton Hall dans le New Jersey, aux États-Unis ${ }^{20}$, s'appuient sur le matériel riche des archives ${ }^{21}$ aussi bien que sur les données de terrain collectées dans les différents pays du continent et en Russie.

2 En prenant pour point de départ la figure historique d'Abraham Petrovitch Hannibal, l'ancêtre d'Alexandre Pouchkine, les contributions nous plongent d'emblée dans l'histoire de la Russie tsariste du XVIII ${ }^{\mathrm{e}}$ siècle avant de s'intéresser aux expéditions des 
Russes en Éthiopie à la fin du XIX ${ }^{\mathrm{e}}$ siècle. Cependant, la trame de l'ouvrage se déroule essentiellement au xx ${ }^{e}$ siècle avec la Révolution bolchévique de 1917 comme point de départ de la consolidation des liens entre la Russie, l'Afrique et le monde noir. Comme le montrent ces intellectuels africains et afro-américains qui ont entrepris le pèlerinage à la « Mecque Rouge » pour y puiser inspiration et idées ou quérir des soutiens pour les luttes de libération nationale et contre le racisme aux États-Unis. Souvent déçus et désillusionnés par l'incapacité des Soviétiques d'intégrer et de reconnaître l'importance de la « race » et de la couleur de la peau dans le discours idéologique, ils ont eu tendance à échanger le communisme contre des aspirations nationalistes et panafricanistes (p. 4).

Pendant la Guerre froide, l'assistance de l'Union soviétique à plusieurs pays du continent (notamment Angola, Mozambique, Afrique du Sud, Ghana, Guinée, Mali, Nigeria, Égypte) dans les domaines militaire, culturel et académique fut importante. Cela a été d'un soutien inestimable aux mouvements de libération, contribuant de facto à l'épilogue du colonialisme et à la construction d'États indépendants. La lutte pour gagner les «cœurs et les esprits » faisait partie des enjeux de cette coopération: des milliers d'Africains sont partis étudier en URSS avec des bourses d'études et ont ouvert aux Soviétiques de nouveaux modes de vie avec les objets de consommation occidentaux - jeans, musique pop et jazz rapportés de vacances passées en Occident ${ }^{22}$. Cependant, le racisme dissimulé dans la rhétorique anticoloniale de la propagande soviétique compliquait le rapprochement: les liens entre la Russie et l'Afrique demeuraient toujours lointainement exotiques tout en se nourrissant des multiples contacts qui s'enracinaient progressivement dans l'histoire complexe de ces échanges. La perestroika, la dissolution de l'URSS et la fin de la Guerre froide ont bouleversé ce processus sans pour autant y mettre fin. Ce fut le début d'une autre époque qu'on ne saurait comprendre sans une analyse approfondie des contacts historiques entre la Russie et les pays du continent africain que ce livre nous restitue de manière remarquable. Les textes réunis présentent, en effet, un projet ambitieux qui se décline en une vision à la fois générale et microscopique des récits historiques et idéologiques des connexions entre la Russie et l'Afrique, mis à l'écart, jusqu'à une date encore récente, de l'analyse de l'histoire globale ${ }^{23}$. Plusieurs disciplines (sociologie, histoire, sciences politiques) s'y côtoient, construisant ainsi une trame de dialogue passionnant autour des récits où s'interjettent marxisme-léninisme, socialisme, capitalisme et racisme sur la toile de fond des mouvements de libération nationale, de la Guerre froide et de la vie soviétique quotidienne. Divisé en deux parties équilibrées et complémentaires, composée chacune de huit chapitres qui traitent des interactions entre la Russie et l'Afrique, le livre fait varier l'angle d'approche géographique selon les parties. Cette composition permet de mettre en évidence à quel point les connexions entre la Russie et l'Afrique ont été nourries par les dynamiques complexes inscrites dans leur propre historicité. Ainsi, lorsque la première partie du livre soulève les thèmes de la présence des Africains ou des Afro-Américains en Russie en évoquant la question du racisme et des idéologies (chapitres 3, 4, 7 et 8), de l'exotisme (chapitres 1, $2,7,8$ ) ou de la constitution des savoirs sur l'Afrique en URSS (chapitres 5 et 6), la seconde partie sur la présence de la Russie en Afrique en affine la compréhension et vient compléter l'analyse des relations entre la Russie et plusieurs pays du continent africain en se focalisant sur les régions de la Corne de l'Afrique (chapitre 10), du sud du continent (chapitres $9,12,14,15$ ) et de l'Afrique de l'Ouest (chapitres 13 et 16). 
C'est dans les deux premiers chapitres que Frances M. Somers Cocks et Allison Blakely ouvrent le débat avec une analyse rétrospective des liens entre la Russie et l'Afrique depuis Pierre le Grand où la première figure historique à s'imposer n'est autre qu'Abraham Petrovitch Hannibal. Ingénieur militaire puis général, Abraham Petrovitch Hannibal est plus connu comme l'arrière-grand-père d'Alexandre Pouchkine, le grand poète russe $\mathrm{du} \mathrm{XIX}^{\mathrm{e}}$ siècle: ses origines sont aujourd'hui disputées entre l'Éthiopie, l'Érythrée et le Cameroun. Ces chapitres donnent à l'ouvrage une tonalité où les Africains apparaissent comme maîtres de leur propre destin en s'engageant pleinement dans les domaines culturels, scientifiques et politiques; et non comme des pions passifs d'une histoire qui les submerge (p. 54). La question de la race, du racisme et des idéologies est un autre fil rouge de cette partie de l'ouvrage. Ainsi, Woodford McClellan dans le chapitre 3 sur l'Université communiste des travailleurs de l'Orient (KUTV), institution mise en place par les bolcheviks afin de former les cadres communistes pour "l'Orient soviétique et étranger» (p. 61), montre comment les Africains et les AfroAméricains venus s'y former réalisaient très vite que le sujet de la couleur de la peau était pratiquement mis à l'écart de la problématique dominante de la lutte des classes, ce qui les condamnait à une existence de citoyens de seconde classe. Les chapitres 7 et 8 prolongent d'une certaine manière cette réflexion en s'intéressant à l'héritage soviétique à travers les traductions du racisme dans la vie quotidienne des Africains et de leurs enfants installés en Russie dans les années 1990-2000. Ils avancent l'hypothèse que ces sentiments "anti-Africains » ont contribué à la création d'une communauté panafricaine à Moscou.

5 Comprendre la manière dont l'Afrique a été pensée en Union soviétique est important pour mieux décrypter les relations entre la Russie et les pays du continent. Les chapitres 5 et 6 interrogent le processus de construction de ce savoir qui n'obéissait pas toujours aux logiques idéologiques ou aux orientations géopolitiques, même si celles-ci n'étaient pas étrangères à sa formulation, comme le montrent Apollon Davidson et Irina Filatova dans le chapitre 5 . Une première phase de cette élaboration des savoirs sur l'Afrique peut ainsi être située avant la révolution d'Octobre 1917 ; elle est surtout liée aux expéditions en Éthiopie de Leonid Artamonov et Alexander Bulatovitch. La seconde période, qui s'étale des années 1920 jusqu'au milieu des années 1950, est associée principalement aux intérêts de l'URSS pour l'Afrique du Sud, renforcés par le fait que le Parti communiste de l'Afrique du Sud était le seul parti communiste en Afrique. Il était représenté au Komintern à partir de 1921. Les bolcheviks voyaient dans les Africains des alliés du prolétariat international dans la lutte contre le capitalisme. Très liée aux activités du Komintern et à son héritage, cette période est tragiquement marquée par les purges staliniennes qui ont fait périr de nombreux chercheurs comme Georgii Gerngros, qui a été exécuté, et Igor Snegirev, mort dans un camp. En dépit de ces accès de "folie » (p.117) et de ces coups d'arrêt, certains chercheurs ont réussi à développer des idées novatrices; telle l'hypothèse d'Ivan Potehin sur le rôle du colonialisme dans la «formation ethnique $»^{24}$. De la fin des années 1950 jusqu'au milieu des années 1980, au gré de l'émergence de nouvelles sources d'information, de l'accès à certaines archives et à la littérature "occidentale » (limité, mais possible), le spectre des problématiques s'élargit considérablement: désormais l'intérêt est porté sur les sujets liés à la libération nationale, aux mouvements religieux, au colonialisme et au post-colonialisme. Cette période est marquée par des productions d'envergure comme l'écriture de l'Encyclopédie d'Afrique $25^{25}$ et de plusieurs ouvrages sur l'histoire de l'Afrique. Cependant, comme le soulignent Apollon Davidson et Irina Filatova, les 
chercheurs soviétiques, s'intéressant aux interactions entre les structures traditionnelles du pouvoir et les autorités coloniales, ont su développer des approches théoriques globalement différentes de celles de leurs collègues occidentaux ${ }^{26}$ (p. 122).

La seconde partie de l'ouvrage met l'accent sur l'historicité et des dynamiques internes des pays du continent. C'est ainsi que Vladimir Shubin, chercheur de l'Institut d'Afrique à Moscou, souligne dans le chapitre 15 sur la politique de l'URSS en Afrique $\mathrm{du}$ Sud que les conflits dans la seconde moitié $\mathrm{du} \mathrm{xx}^{\mathrm{e}}$ siècle dans diverses régions ont été principalement animés par les dynamiques internes de la région et non pas par la confrontation entre superpuissances sur le continent. Auparavant, dans le chapitre 14, Olayiwola Abegunrin montre que les approvisionnements en matériel militaire et ressources humaines, l'envoi de conseillers militaires en Angola, au Mozambique, en Namibie et en Afrique du Sud, ont façonné des liens tangibles entre l'Union soviétique et les mouvements de libération nationale entre 1960 et 1990. Cependant, l'URSS n'a pas tiré profit de cette occasion pour parvenir à battre en brèche l'influence de l'Occident dans la région. Constat que Sergey Mazov fait déjà dans le chapitre précédent. En croisant les archives soviétiques et américaines ${ }^{27}$, il en vient en effet à la conclusion que dans trois pays de la région de l'Afrique de l'Ouest (Guinée, Ghana et Mali), malgré les efforts de la propagande et nonobstant des moyens matériels considérables, l'URSS n'est pas arrivée à asseoir véritablement son influence. Ce qui ouvre à la perception que les Africains pouvaient avoir des Soviétiques dans les chapitres 12 et 16, rédigés respectivement par Thomas Burgess et Maxim Matusevich. Deux exemples leur servent de fil conducteur : le premier concerne les idées socialistes d'Ali Sultan Issa à Zanzibar et leur impact, le second analyse l'image de l'URSS, de sa grandeur industrielle et géopolitique au Nigéria. Les Nigérians appréciaient les réalisations soviétiques, telles que l'industrialisation rapide ou la gratuité des soins de santé et de l'éducation, mais avaient tendance à rejeter le reste du « kit » à caractère idéologique. Comme dans les autres pays du continent, les Nigérians n'ont eu de cesse de comparer les modèles soviétiques et occidentaux pour utiliser le « meilleur des deux mondes" en fonction de leurs propres objectifs, comme le prouve l'histoire de la coopération nigériano-soviétique pendant la guerre du Biafra (1967-1970).

7 Basé sur le croisement d'archives et de perspectives théoriques de chercheurs venant d'horizons et d'héritages disciplinaires différents, cet ouvrage constitue une importante contribution à la déconstruction de la «bibliothèque coloniale » - tâche qui semble impossible à réaliser sans prendre en compte les liens historiques profonds entre l'URSS/Russie et l'Afrique. De ce point de vue, Africa in Russia. Russia in Africa constitue indubitablement une base pour des recherches ultérieures dépassant largement le champ des "area studies» et s'inscrivant dans les interrogations sur la portée du projet sociétal soviétique appliqué à l'Afrique. 


\section{NOTES}

20. Avant de partir aux États-Unis, Maxim Matusevich a vécu à Saint-Pétersbourg, sa ville d'origine.

21. Il s'agit surtout des archives du ministère des Affaires étrangères de la Fédération de Russie (AVPRF) et des Archives d'État d'Histoire contemporaine (RGANI) à Moscou.

22. M. Matusevich, «An Exotic Subversive : Africa, Africans and the Soviet Everyday ", Race \& Class, 49 (4), 2008, pp. 57-81.

23. Il faut prendre ici en considération le projet de recherche ÉLITAF (Étudiants et élites africaines formés dans les pays de l'ex-bloc soviétique) mis en place en 2012 par les membres coordinateurs du Réseau international Afrique Monde, avec le soutien de la FMSH et de l'IRIS.

24. I. Pотенin, Formirovanie natsionalnoi obshchnosti yuzhnoafricanskikh bantu (La formation de la communauté bantou en Afrique du Sud), Moscou, Izd-vo Akademii Nauk SSSR, 1955.

25. Afrika. Entsiklopedia (Afrique: Encyclopédie), v. 1-2, Moscou, Nauka, 1986-1987.

26. Pour étayer leurs propos, les auteurs citent différents travaux: I. S LEDZEVSKIY, Khausanskiie emiraty Severnoi Nigerii (Les émirats hausa au Nord du Nigeria), Moscou, Nauka, 1974 ; I. Filatova, Novaya i noveyshaya istoriia Kenii (L'histoire contemporaine du Kenya), Moscou, Nauka, 1985. Malheureusement, ils ne développent pas davantage l'idée de l'originalité des approches.

27. Il s'agit des Archives du ministère des Affaires étrangères de la Fédération de la Russie (AVPRF), des Archives d'État d'Histoire contemporaine (RGANI) et des Archives nationales des États-Unis (US National Archives). 